\title{
Effect of cationic surfactant on the adsorption characteristics of anionic surfactant on cellulose surface
}

\author{
Santanu Paria, C. Manohar, Kartic C. Khilar* \\ Department of Chemical Engineering, Indian Institute of Technology, Bombay Powai, Mumbai 400076, India
}

\begin{abstract}
Adsorption kinetics and equilibrium studies of sodium dodecylbenzenesulfonate (NaDBS) on cellulosic surface are investigated. Modifications in adsorption induced by cationic surfactant cetyltrimethylammonium bromide (CTAB) are studied. It is concluded that the rate of adsorption and the amount of adsorption are increased. Importance of these observations in detergency is indicated.
\end{abstract}

Keywords: Adsorption enhancement; Mixed surfactant; Ion pair; Cellulose-water interface

\section{Introduction}

Adsorption of surfactant sodium dodecylbenzenesulfonate $(\mathrm{NaDBS})$ is of great interest in detergency because this happens to be the key ingredient in majority of the detergent formulations [1]. Amount of surfactant adsorbed has a direct bearing on the cleanliness of the fabrics and methods of enhancing this adsorption would be most welcome.

One of the methods of achieving this is by incorporating other surfactants in to the formulation [2]. Designing mixed surfactant systems for applications has been mostly by trial and error [3] and in the literature mostly anionic-nonionic surfactant mixtures have been investigated [4-8]. The studies on cationic-anionic surfactant mixtures are very few in view of the 'fear' of precipitation. Huang et al. [9] have investigated the adsorption of cationic surfactant on silica surface from a mixture of anionic-cationic mixture. They observed that amount of cationic adsorbed was enhanced by the presence of anionic surfactant. Patist et al. [10] have studied adsorption from mixtures and have proposed that at 3:1 and 1:3 compositions compact hexagonal structural arrangements are formed on the interface.

In the present paper we investigate the effect of cetyltrimethylammonium bromide (CTAB) adsorption of NaDBS on cellulosic surface. This study is of interest in

\footnotetext{
* Corresponding author. Fax: +91-22-2572-68-95.

E-mail address: kartic@che.iitb.ac.in (K.C. Khilar).
}

detergency from two points of view. Cationic surfactants are known to be bactericides [11] and cloth softeners [12] and properly designed incorporation of these two characteristics in to a detergent formulation would be of great interest.

\section{Experimental}

\subsection{Materials}

Anionic surfactant, sodium dodecylbenzenesulfonate was obtained from Fluka Chemicals. Nonionic surfactant, polyoxyethylene (number of segments 9.5) glycol tert-octylphenyl ether (Triton X-100 or TX-100) was obtained from Sigma. NaDBS and TX-100 were used as received, without any further purification. Cationic surfactant, cetyltrimethylammonium bromide was obtained from Ranbaxy Fine Chemicals Ltd., India. It was recrystallized twice from an acetone:methanol (3:1) mixture before use [13]. Adsorbent used was a Whatman-40 ash less filter paper (9 $\mathrm{cm}$ diameter) from Whatman International Ltd., UK. The BET multipoint surface area $\left(\mathrm{N}_{2}\right.$ adsorption) was $16.5 \mathrm{~m}^{2} / \mathrm{g}$. Double distilled water of pH 5.6 and conductivity $1.2 \mu \mathrm{S}$ ( $\mu$ Mho) was used for the experiment.

\subsection{Methods}

The filter paper was washed thoroughly with double distilled water to remove the dust and soluble ions from the 
filter paper till the conductivity of the washed water became equal to that of the distilled water. Then the filter paper was dried in oven for $1.5-2 \mathrm{~h}$ at around $50^{\circ} \mathrm{C}$ until the weight of the filter paper became constant. The concentration of NaDBS was determined by measuring UV absorbance at $223 \mathrm{~nm}$ wavelength using UV-Vis spectrophotometer (Shimadzu, UV-160A model). Quartz glass cells (Hellma) of $10 \mathrm{~mm}$ path length were used. The surface tension was measured using a Du-Noüy ring tensiometer (Fisher surface tensiomat, Model 21). Conductivity was measured by an auto ranging conductivity meter (Equiptronics, Mumbai, India) using cell constant, $k=1$.

For the adsorption study, a single surfactant solution of desired concentration was prepared by diluting a concentrated stock solution. Calculated amount of the second surfactant was added to this solution $12 \mathrm{~h}$ prior to each adsorption experiment for equilibration. For each of experiment, a $0.580 \mathrm{~g}$ of filter paper was used after cutting into small pieces. A $10 \mathrm{ml}$ surfactant solution was used for each of experiment. The system was stirred slowly by shaking the glass bottles. All the experiments were done at $25^{\circ} \mathrm{C}$. The experiments were repeated at least three times and the average data were plotted.

Adsorption measurements were carried out at five different mixing ratios, 50:1, 25:1, 20:1, 15:1 and 10:1 (moles of anionic:moles of cationic). The surfactant concentrations were chosen below the CMC of the individual and mixed surfactant systems. In anionic-cationic mixtures there was a tendency to form precipitate and this was avoided by working below the CMC, where it is possible to study in a wide range of mixing ratios.

Evaporation studies were carried out by taking $10 \mathrm{ml}$ of the solutions in identical beakers and keeping them in a closed area to minimize the effect of dust. The temperature of the room was maintained to $25^{\circ} \mathrm{C}$. Weight loss by evaporation was measured by monitoring initial and final weight at the desired time.

\section{Result and discussions}

\subsection{Kinetic measurements}

Fig. 1 indicates the amount of NaDBS adsorbed at any given time during the experiment keeping the NaDBS amount constant at $0.2 \mathrm{mM}$. The results are in terms of the $\mathrm{mg}$ of NaDBS adsorbed per gram of filter paper. These experiments were carried out under three types of conditions.

1. Both NaDBS and CTAB were mixed to obtain concentrations of 0.2 and $0.02 \mathrm{mM}$, respectively, in the solution for adsorption.

2. CTAB was pre-adsorbed (using a $0.02 \mathrm{mM}$ solution) on filter paper, washed once, before NaDBS was allowed to adsorb.

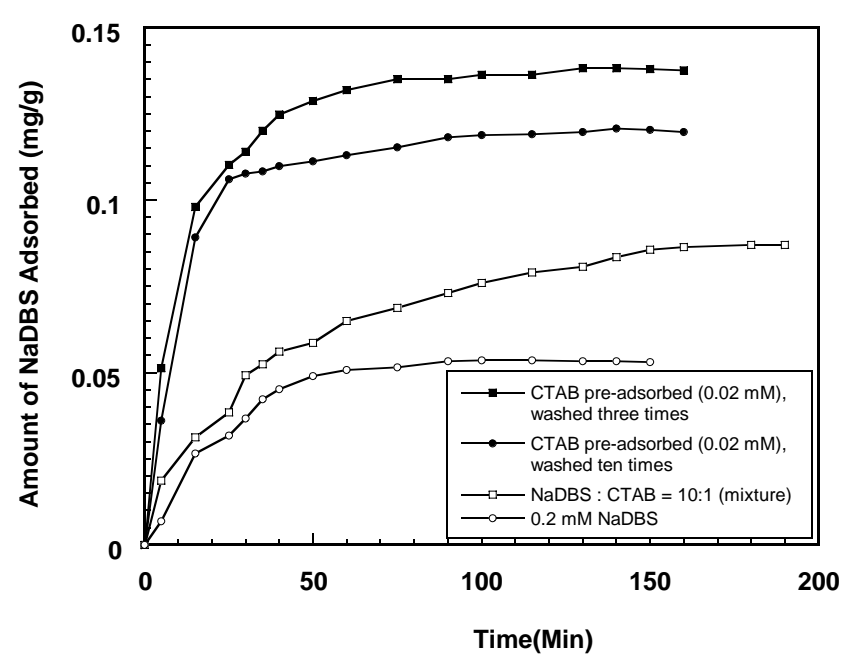

Fig. 1. Comparison of adsorption enhancement between NaDBS $(0.2 \mathrm{mM})$, NaDBS/CTAB mixture (10:1 mole ratio) and NaDBS (0.2 mM) with pre-adsorbed filter paper in $0.02 \mathrm{mM} \mathrm{CTAB}$ solution.

3. The CTAB pre-adsorbed filter paper was washed 10 and 3 times with distilled water prior to adsorption from NaDBS solution.

In the case of NaDBS only, the adsorption is weak. The reason, most probably, is because the cellulosic surface is expected to be negatively charged $(\sim-28 \mathrm{mV})[14]$ and the adsorption of the negatively charged head group of the surfactant is opposed. The adsorption of NaDBS takes place mostly at hydrophobic sites.

CTAB has a positively charged head group and this, on adsorption, is expected to convert the negatively charged site to a hydrophobic site. This expectation is confirmed by pretreating the surface by CTAB solution. It is noticed that the rate of adsorption and also the amount adsorbed at equilibrium is enhanced. It is interesting to notice that these

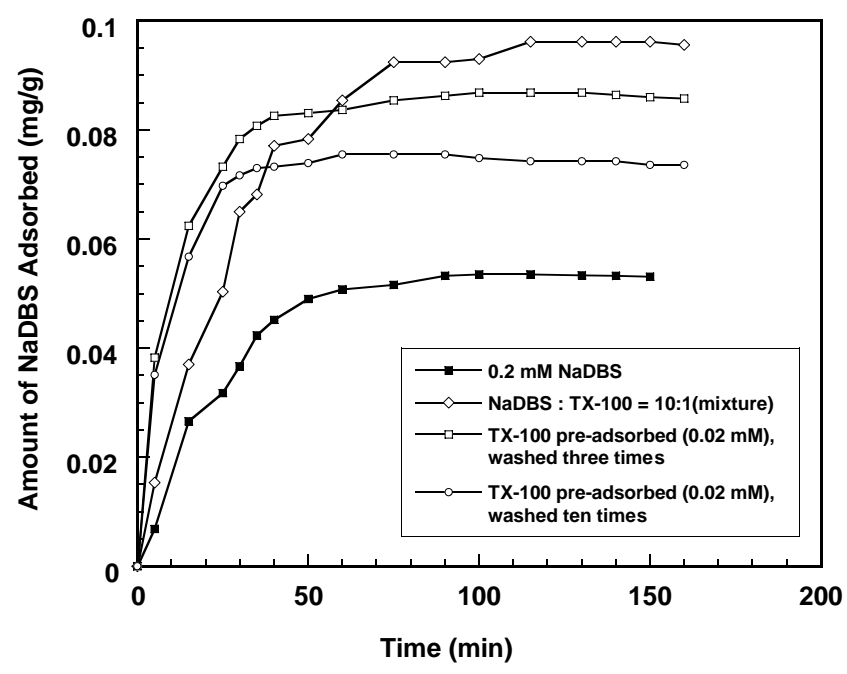

Fig. 2. Comparison of adsorption enhancement between NaDBS $(0.2 \mathrm{mM})$, NaDBS/TX-100 mixture (10:1 mole ratio) and NaDBS $(0.2 \mathrm{mM})$ with pre-adsorbed filter paper in $0.02 \mathrm{mM}$ TX-100 solution. 


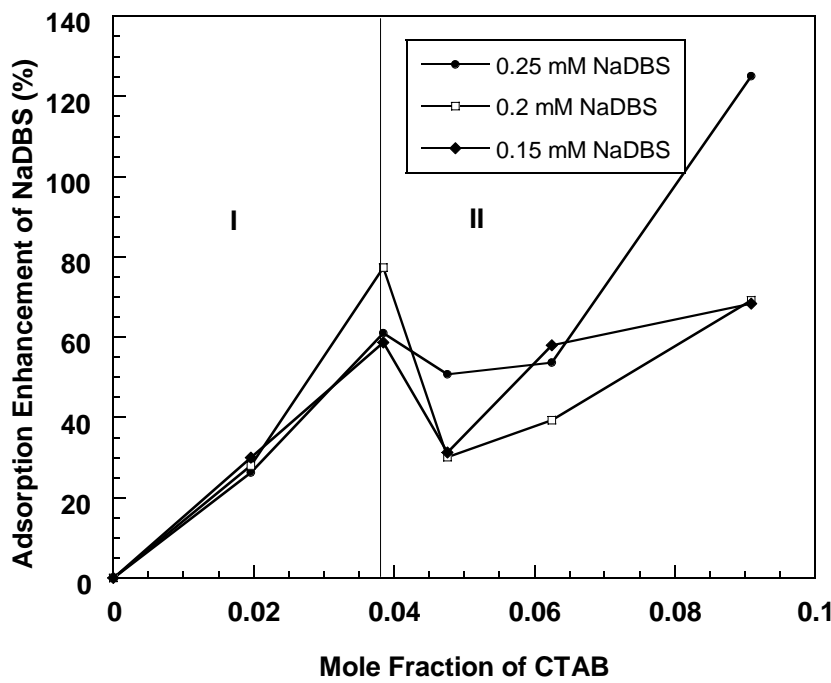

Fig. 3. Adsorption enhancement $(E)$ of NaDBS at different concentration of CTAB.

enhancements remained even after washing the filter paper 10 times.

This aspect has practical implications. Cationic surfactants are used as cloth softeners it would be interesting to check whether the efficiency of detergency, in the subsequent washings, after softening would be enhanced.

Fig. 1 also indicates that the enhancement in adsorption after mixing the two surfactants NaDBS and CTAB in the solution. The enhancements both in rate and amount are smaller compared to the pretreated surfaces. The main reason for this difference is because cationic and anionic surfactants, in mixture, form an ion pair and this behaves like surfactant with almost no charge and hence lesser adsorption than CTAB. The adsorption appears to be purely due to hydrophobic interactions. This view is confirmed by the adsorption studies carried out using a nonionic surfactant Tri-

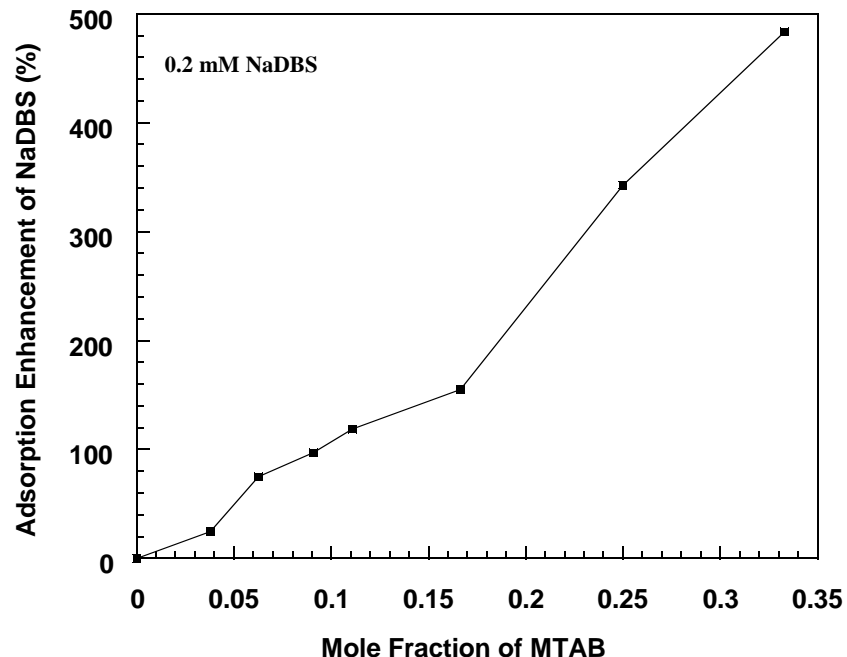

Fig. 4. Adsorption enhancement $(E)$ of NaDBS at different concentration of MTAB.

ton X-100 (TX-100). The results are shown in Fig. 2. It is interesting to notice that the enhancement in adsorption for 10:1 ratio of NaDBS:TX-100 (same concentration as that for CTAB in Fig. 1) is approximately same and washing and pretreatment as done previously gives approximately same result.

The important message we get from these studies is that, to enhance the adsorption of NaDBS, it is better to pretreat the surface with CTAB than mixing the same in formulation. In the latter case the ion pair formation hinders the adsorption at charged sites.

\subsection{Equilibrium studies}

These studies were carried out to quantify the enhancement in the adsorption of NaDBS by the other surfactant

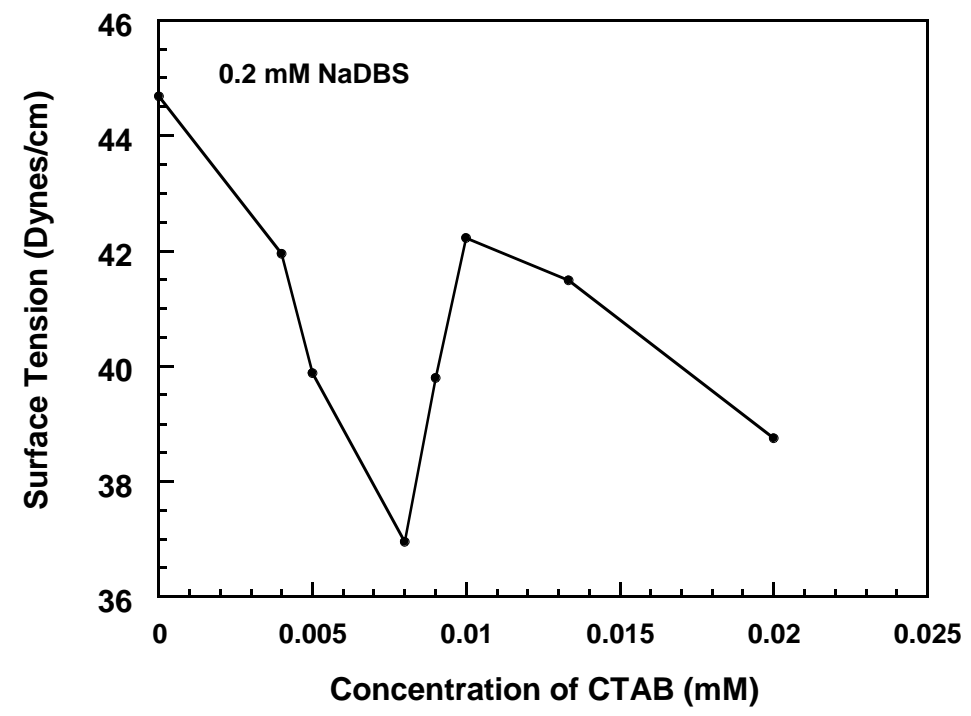

Fig. 5. Plot of surface tension of NaDBS/CTAB mixture at different NaDBS/CTAB mixing ratio. 
such as CTAB. In order to facilitate this aspect we define a quantity 'enhancement factor', $E$

$E=\frac{\Gamma_{\mathrm{mix}}-\Gamma_{\mathrm{NaDBS}}}{\Gamma_{\mathrm{NaDBS}}} \times 100$

which measures in percentage the efficiency of enhancement. Where $\Gamma_{\mathrm{NaDBS}}$ and $\Gamma_{\text {mix }}$ are the equilibrium amounts adsorbed in $\mathrm{mg} / \mathrm{g}$ of $\mathrm{NaDBS}$ from solution containing only NaDBS and from solution containing mixture, respectively.

Fig. 3 shows the enhancement in adsorption induced by CTAB at three different concentrations $0.15,0.2$ and $0.25 \mathrm{mM}$ of NaDBS. The enhancement observed is not just linear but shows peak at a mole fraction of 0.04 of CTAB. Such observations in other systems have been attributed to formation of closely packed hexagonally ordered structures on the surface of the adsorbent [10]. The peak at 0.04 mole fraction does not correspond to closely packed structures and at present we do not have any explanation. However this indicates that if one has to incorporate CTAB in to a formulation the optimum concentration is at 0.04 mole fraction.

In order to check if this value of 0.04 is related to head group interactions we have performed an experiment with $\mathrm{C}_{14} \mathrm{TAB}$ (MTAB), where the head group is same as CTAB but the chain length is smaller. Fig. 4 shows no peak in $E$ versus mole fraction indicating that the phenomenon is dependent on hydrophobic part too.

Fig. 5 shows the effect of the CTAB addition on surface tension at a fixed concentration of $0.2 \mathrm{mM}$ of $\mathrm{NaDBS}$. This also shows a dip at 0.04 ratio and indicates that area per molecule shows a minimum.

\section{Conclusions}

Anionic-cationic mixtures are of interest from the point of view of their use in detergency. The adsorption studies indicate that in cellulosic surfaces there are both hydrophobic and electrically charged sites with negatively charge.
Pretreating the surface with a cationic appears to enhance both the rate and amount of adsorption than using a mixture of cationic-anionic surfactants in the formulation. A mole fraction of 0.04 of CTAB appears to be optimum value if one has to use mixtures of surfactants. This value of 0.04 is dependent on the chain length of the surfactant.

\section{Acknowledgements}

The authors wish to thank Drs. V.R. Dhanuka, Girish Rao and Peter Garrett of Unilever Research Center, India, for many use full discussions on this topic. The authors wish to thank Unilever Research Center, India, for providing the financial support for this work.

\section{References}

[1] N. William, Fundamentals of Detergency, Reinhold, New York, 1950.

[2] S. Verma, V.V. Kumar, J. Colloid Interf. Sci. 1 (1998) 207.

[3] A. Shiloach, D. Blankschtein, Langmuir 14 (1998) 1618.

[4] P. Somasundaran, L. Huang, Polish J. Chem. 71 (1997) 568.

[5] Y. Gao, C. Yue, S. Lu, W. Gu, T. Gu, J. Colloid Interf. Sci. 100 (1984) 581.

[6] P. Somasundaran, E. Fu, Q. Xu, Langmuir 8 (1992) 1065.

[7] J.F. Scamehorn, R.S. Schechter, W.H. Wade, J. Colloid Interf. Sci. 85 (1982) 494.

[8] Q. Xu, T.V. Vasudevan, P. Somasundaran, J. Colloid Interf. Sci. 142 (1991) 528.

[9] Z. Huang, Z. Yan, T. Gu, Colloid Surf. 36 (1989) 353.

[10] A. Patish, S. Devi, D.O. Shah, Langmuir 15 (1999) 7403.

[11] H.T. Patterson, T.H. Grindstaff, Surface characteristics of fibers and textiles, Part II, in: M.J. Shick (Ed.), Marcel Dekker, New York, 1977, Chapter 12, p. 448

[12] D.N. Rubingh, Cationic surfactants-physical chemistry, in: D.N. Rubingh, P.M. Holland (Eds.), Marcel Dekker, 1991, Chapter 10, p. 469.

[13] T.R. Desai, S. Dixit, J. Colloid Interf. Sci. 179 (1996) 544.

[14] H. Schott, Detergency theory and test methods, Part 1, in: W.G. Cutler, R.C. Davis (Eds.), Marcel Dekker, New York, 1972 (Chapter 6). 